\title{
Hubcap and ignition switch designs - case studies in Independence Axiom
}

\author{
Hilario (Larry) $\mathrm{Oh}^{1, *}$ \\ ${ }^{1}$ MIT Park Center for Complex Systems, Cambridge, MA, USA
}

\begin{abstract}
Independence Axiom offers designers a guide to good design. It declares that the design parameters (DPs) conceived for a good design must maintain the independence of the design functional requirements (FRs). Specifically, by relating FRs to DPs through a design matrix $[\mathrm{DM}]$ with elements $\partial \mathrm{FR} / \partial \mathrm{DP}$, Independence Axiom declares that only designs with diagonal or triangular design matrix can maintain the functional independence of FRs; and that they should be the only acceptable ones.

Starting with the formal definition of functional independence, we derive the criterion for functional independence of FRs as the Jacobian determinant $|\mathrm{J}| \neq 0$; where the Jacobian matrix $[\mathrm{J}]$ is shown to be identically equal to [DM]. We further show that if and only if $|\mathrm{J}| \neq 0$ can the design FRs achieve their target values. Thus the criterion $|\mathrm{J}| \neq 0$ substantiates the declaration of Independence Axiom since determinant of a diagonal or triangular design matrix is not equal to zero. It serves as the mathematical basis for teaching and implementing Independence Axiom in design.
\end{abstract}

Two case studies are presented to illustrate the implementation of Independence Axiom via the Jacobian determinant I J I.

\section{Introduction}

Prior to the introduction of the Independence Axiom, there was no scientific basis to assess the goodness of a design. Assessment is usually done through build and test. In 1979, Independence Axiom was introduced to define goodness of a design and to guide designers through the design process. The axiom is assumed to be self-evident truth for which there is no exception or counter-example. It cannot be proven nor derived from laws of nature [1].

Because of the axiomatic assumption it imposes, Independence Axiom has been rejected by part of the design community. To satisfy the critics and to further advance Independence Axiom as a science in design, we develop in Section 2 a mathematical basis to support the axiomatic assumption. This is followed by two case studies in Section 3. The hubcap case heightens the need for functional independence when functional requirements are opposing. The ignition switch case is a return of the hubcap problem. It is presented to stress the point that design as a science - e.g., Independence Axiom - should be taught so that design mistakes need not be learned again and again.

\section{Math basis of Independence Axiom}

Usually, a design has multiple functional requirements FRs. These FRs are realized with design solutions that involve physical entities, which we label as DPs the design parameters. The FRs are related to DPs through physical laws which we denote as $\mathrm{f}_{\mathrm{k}}(\cdot), \mathrm{k}=1,2, \cdots, \mathrm{n}$ :

\footnotetext{
*Corresponding author: ohlarry@yahoo.com
}

$$
\begin{gathered}
\mathrm{FR}_{1}=\mathrm{f}_{1}\left(\mathrm{DP}_{1}, \cdots, \mathrm{DP}_{\mathrm{m}}\right) \\
\vdots \\
\mathrm{FR}_{\mathrm{n}}=\mathrm{f}_{\mathrm{n}}\left(\mathrm{DP}_{1}, \cdots, \mathrm{DP}_{\mathrm{m}}\right)
\end{gathered}
$$

Or in vector form,

$$
\mathbf{F R}=\mathbf{f}(\mathbf{D P})
$$

In the above and hereafter, bolded quantities denote

vectors, bracketed quantities denote matrices and $\mathbf{f}(\cdot)$ denotes vector-valued functions.

Axiomatic Design offers designers two axioms to guide them in the choice of design solutions [1]. In one of the axioms, the Independence Axiom, it directs designers to go for solutions with DPs that maintain the independence of FRs. Specifically, Independence Axiom proposes an equation in the form:

$$
\mathbf{F R}=[\mathrm{A}] \mathbf{D P}
$$

where elements of the [A] matrix, the design matrix, are:

$$
A_{i j}=\frac{\partial F R_{i}}{\partial D_{j}}
$$

Considering only those elements " $\mathrm{X}$ " that have a strong effect on an FR relative to its tolerance, Independence Axiom declares that only designs with diagonal or triangular design matrix $[\mathrm{A}]$ in " $\mathrm{X}$ " can maintain the functional independence of FR. They are therefore the acceptable ones. The mathematical basis for such a declaration has been derived in [2]. It is further reviewed here as a lead up to the case studies that follow. 
The task in design is to find the root of the equation:

$$
\mathbf{F R}-\mathbf{F R}^{*}=\mathbf{f}(\mathrm{DP})-\mathbf{F R}^{*}=\mathbf{0}
$$

that enables $\mathbf{F R}$ to achieve its target value $\mathbf{F R}^{*}$.

Usually $\mathbf{f}(\mathbf{D P})$ is nonlinear so that the $\operatorname{root} \equiv \mathbf{f}^{-1}\left(\mathbf{F R} \mathbf{R}^{*}\right)$ cannot be solved analytically. In its place, $\mathbf{f}(\mathbf{D P})$ in Equation (1) is linearized via Taylor expansion:

$$
\mathbf{f}(\mathbf{D P})-\mathbf{F R}^{*} \approx \mathbf{f}\left(\mathbf{D} \mathbf{P}^{\mathrm{k}}\right)+[\mathrm{J}]\left(\mathbf{D P}-\mathbf{D P} \mathbf{P}^{\mathrm{k}}\right)-\mathbf{F} \mathbf{R}^{*}=\mathbf{0} .
$$

Above equation is then solved iteratively via a succession of linear equations:

$$
\mathbf{D} \mathbf{P}^{\mathrm{k}+1}=\mathbf{D} \mathbf{P}^{\mathrm{k}}-[\mathrm{J}]^{-1}\left\{\mathbf{f}\left(\mathbf{D} \mathbf{P}^{\mathrm{k}}\right)-\mathbf{F} \mathbf{R}^{*}\right\} .
$$

Iteration with $\mathrm{k}=0,1,2, \ldots$ ends when $\left\|\mathbf{D} \mathbf{P}^{\mathrm{k}+1}-\mathbf{D} \mathbf{P}^{\mathrm{k}}\right\|$ is less than a desired accuracy. At that point $\mathbf{D P}^{\mathrm{k}+1}$ serves as the root to Equation (1).

In the above, the matrix $[\mathrm{J}]$ is the Jacobian of $\mathbf{f}(\mathbf{D P})$ with element $\partial \mathrm{FR}_{\mathrm{i}} / \partial \mathrm{DP} \mathrm{p}_{\mathrm{j}}$ evaluated at $\mathbf{D P} \mathbf{P}^{\mathrm{k}}$. It is identically equal to $\mathrm{A}_{\mathrm{ij}}$, the element of the design matrix [A] defined earlier. If Jacobian determinant $|\mathrm{J}|=0$, then the inverse of the Jacobian $[\mathbf{J}]^{-1}$ does not exist; iterative root finding cannot proceed and FR cannot achieve its target FR*. This is the crux of design's failure to achieve its target.

Under what condition then is $|\mathbf{J}|=0$ ? In the next section, we show this condition to be the consequence of functional dependency among FRs. While the derivation is based on two FRs involving two DPs, similar derivation holds true for $n$ FRs involving $n$ DPs, $n>2$.

We start with the formal definition of functional dependency. Namely, $\mathrm{FR}_{2}$ is functionally dependent on $\mathrm{FR}_{1}$ if it is a function of $\mathrm{FR}_{1}$ :

$$
\mathrm{FR}_{2}=\mathrm{FR}_{2}\left(\mathrm{FR}_{1}\right)
$$

Applying the chain rule for differentiation, we have

$$
\begin{aligned}
& \frac{\partial \mathrm{FR}_{2}}{\partial \mathrm{DP}_{1}}=\frac{\partial \mathrm{FR}_{2}}{\partial \mathrm{FR}_{1}} \cdot \frac{\partial \mathrm{FR}_{1}}{\partial \mathrm{DP}_{1}} \\
& \frac{\partial \mathrm{FR}_{2}}{\partial \mathrm{DP}_{2}}=\frac{\partial \mathrm{FR}_{2}}{\partial \mathrm{FR}_{1}} \cdot \frac{\partial \mathrm{FR}_{1}}{\partial \mathrm{DP}_{2}}
\end{aligned}
$$

Subtract $\frac{\partial \mathrm{FR}_{1}}{\partial \mathrm{DP}_{1}} \times$ Equation (3) from $\frac{\partial \mathrm{FR}_{1}}{\partial \mathrm{DP}_{2}} \times$ Equation (2) to eliminate the product $\left(\frac{\partial \mathrm{FR}_{2}}{\partial \mathrm{FR}_{1}}\right)\left(\frac{\partial \mathrm{FR}_{1}}{\partial \mathrm{DP}_{2}}\right)\left(\frac{\partial \mathrm{FR}_{1}}{\partial \mathrm{DP}_{1}}\right)$, we arrive at the condition for functional dependency:

$$
\left(\frac{\partial \mathrm{FR}_{1}}{\partial \mathrm{DP}_{2}}\right)\left(\frac{\partial \mathrm{FR}_{2}}{\partial \mathrm{DP}_{1}}\right)-\left(\frac{\partial \mathrm{FR}_{1}}{\partial \mathrm{DP}_{1}}\right)\left(\frac{\partial \mathrm{FR}_{2}}{\partial \mathrm{DP}_{2}}\right)=0
$$

Note that $\left(\frac{\partial F R_{2}}{\partial D P_{2}} \frac{\partial F R_{1}}{\partial D P_{1}}-\frac{\partial F R_{1}}{\partial D P_{2}} \frac{\partial F R_{2}}{\partial D P_{1}}\right)$ is the determinant

$$
\left|\begin{array}{ll}
\frac{\partial \mathrm{FR}_{1}}{\partial \mathrm{DP}_{1}} & \frac{\partial \mathrm{FR}_{1}}{\partial \mathrm{DP}_{2}} \\
\frac{\partial \mathrm{FR}_{2}}{\partial \mathrm{DP}_{1}} & \frac{\partial \mathrm{FR}_{2}}{\partial \mathrm{DP}_{2}}
\end{array}\right| \equiv|\mathrm{J}| .
$$

Thus, if $\mathrm{FR}_{2}$ is functionally dependent on $\mathrm{FR}_{1}$, then

$$
|\mathrm{J}|=0 \text {. }
$$

The converse case also holds true, [2]. If $|\mathrm{J}|=0$, then

$$
\mathrm{FR}_{2} \text { is functionally dependent on } \mathrm{FR}_{1} \text {. }
$$

Combining the above two statements,

If and only if $|\mathrm{J}|=0$, then FRs are functionally dependent;

So that $\left(\mathrm{FR}_{1}, \mathrm{FR}_{2}\right)$ cannot achieve the targets $\left(\mathrm{FR}_{1}^{*}, \mathrm{FR}_{2}^{*}\right)$

By the same token,

If and only if $|\mathrm{J}| \neq 0$, then FRs are functionally independent;

and $\left(\mathrm{FR}_{1}, \mathrm{FR}_{2}\right)$ can achieve targets $\left(\mathrm{FR}_{1}^{*}, \mathrm{FR}_{2}^{*}\right)$.

As noted earlier, the design matrix $[\mathrm{A}]$ is identical to the matrix $[\mathrm{J}]$. Its determinant for a diagonal or triangular [A] is not equal to zero. Thus according to the statement above, the FRs are functionally independent and the FR can achieve its target value FR *. In other words, Independence Axiom is corroborated by the condition $|\mathrm{J}| \neq 0$. The $|\mathrm{J}|$ criterion thus can serve as the basis to verify compliance with the Independence Axiom.

\section{Case studies in Independence Axiom}

\subsection{Hubcap - a functionally dependent design}

This case study was described in [3]. It is reviewed here to elucidate the functional dependency of the design. Figure 1A shows the front view of GM 1986-88 Pontiac 6000 hubcap. Figure 1B is its back view showing three pairs of clips spaced $120^{\circ}$ apart such that the 6 clips form a circle of diameter $D_{\text {clip }}$. Figure $1 \mathrm{C}$ is an enlarged view of the pair of clips at the 4 o'clock position. The clips are cantilevers fixed at a post. Figure 1D shows the wheel rim with a circumferential ledge, shown in white, onto which the hubcap snapped on. The diameter of the ledge 
$\mathrm{D}_{\text {rim }}$ is smaller than the clip diameter $\mathrm{D}_{\text {clip }}$. As the hubcap is snapped on to the rim, the ledge catches the cantilever clips. Wheel retention force is developed via interference fit $=\mathrm{k} \delta$; where $\mathrm{k}$ is the spring rate of the cantilever and $\delta$ is the interference $=\left(D_{\text {clip }}-D_{\text {rim }}\right) / 2$

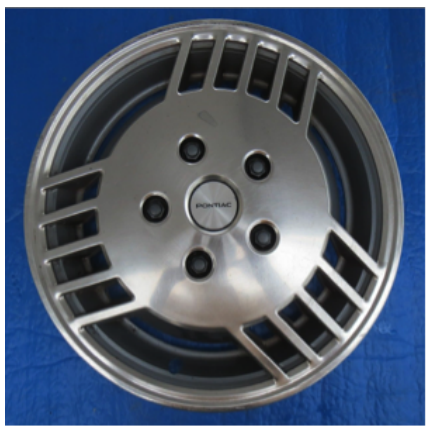

(A) Hubcap front

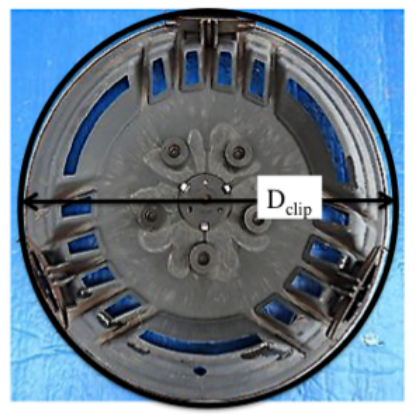

(B) Hubcap back
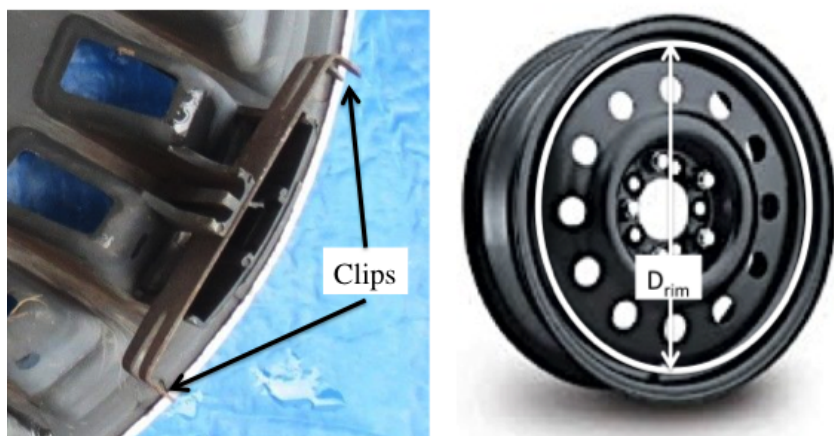

(C) Cantilever clips
(D) Wheel rim

Fig. 1. Attachment of hubcap to wheel rim

There are two FRs for the hubcap design.

$\mathrm{FR}_{1}=$ retain hubcap on cornering and over road bumps, the larger the retention force the better;

$\mathrm{FR}_{2}=$ make hubcap removal easier during flat tire repair, the smaller the retention force the better.

Because the two FRs are opposing, the larger the better versus the smaller the better, it is crucial that the design solution adopted can satisfy both FRs independent of each other. If not, there would be conflict. In the above design, the solution adopted is interference fit. So that both FRs are a function of the same variables $(\mathrm{k}, \delta)$ :

$$
\mathrm{FR}_{1}=\mathrm{FR}_{2}=\mathrm{k} \delta .
$$

The two FRs are functionally inter-dependent which we confirm by checking if $|\mathrm{J}|=0$ :

$$
\begin{gathered}
{\left[\begin{array}{c}
\mathrm{FR}_{1} \\
\mathrm{FR}_{2}
\end{array}\right]=\left[\begin{array}{c}
\mathrm{k} \delta \\
\mathrm{k} \delta
\end{array}\right] \cdot} \\
{[\mathrm{J}]=\left[\begin{array}{cc}
\frac{\partial \mathrm{FR}_{1}}{\partial \mathrm{k}} & \frac{\partial \mathrm{FR}_{1}}{\partial \delta} \\
\frac{\partial \mathrm{FR}_{2}}{\partial \mathrm{k}} & \frac{\partial \mathrm{FR}_{2}}{\partial \delta}
\end{array}\right]=\left[\begin{array}{cc}
\delta & \mathrm{k} \\
\delta & \mathrm{k}
\end{array}\right]}
\end{gathered}
$$

$$
|\mathrm{J}|=\left|\begin{array}{cc}
\delta & \mathrm{k} \\
\delta & \mathrm{k}
\end{array}\right|=\delta \mathrm{k}-\delta \mathrm{k}=0 .
$$

This confirms the design violates Independence Axiom.

To recap, although we set out to define two functionally independent FRs, the solution we choose interference fit - does not fulfill the intention. It induces conflict between them. Any attempt to satisfy one FR invariably dissatisfies the other. Simply put: one DP, interference fit, cannot satisfy two FRs, retention and removal. The consequence was $25 \%$ of hubcaps fell off as the car corners or hits bumps and potholes. And some customers complained about the difficulty in removing the hubcap for a flat tire repair.

Back in 1986, we had to resort to a compromised target value of $F R=32 \mathrm{~N}$; and reduce variability around it via robust design optimization as follows, see [4].

Define a (compromised) target: $\mathrm{FR}_{\text {target }}$;

Identify "noise", the source of variability: $\delta$;

Minimize $\partial \mathrm{FR} / \partial \delta(=\mathrm{k})$ sensitivity to noise: $\mathrm{k} \rightarrow$ small;

Subject to the constraint that: $\mathrm{k} \delta=\mathrm{FR}_{\text {target }}$.

The results of the optimization are shown in Figure 2. The solution was a compromise. It had limited success because the performance of an ill-conceived design cannot be improved via subsequent optimization.

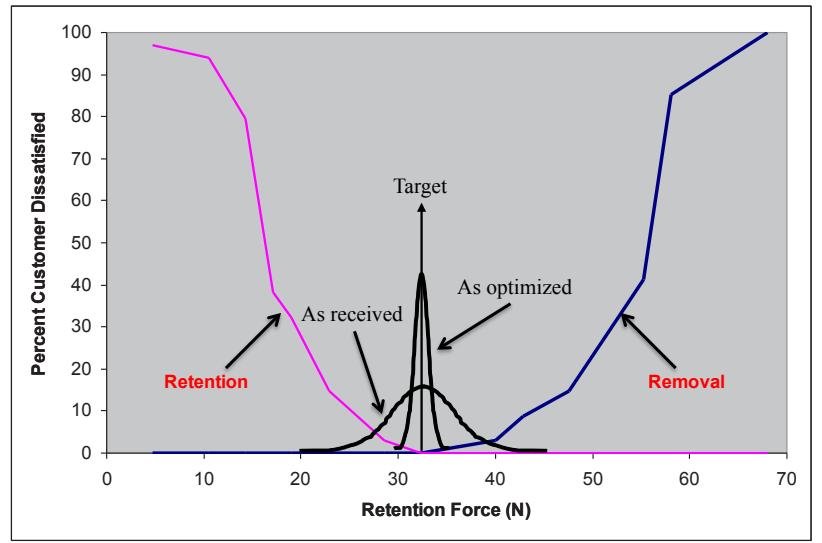

Fig. 2. Customer dissatisfaction versus retention force

In the next case study, we show that the same mistake was committed - coupling of opposing FRs. We further show how to resolve the coupling thereby avoid conflict.

\subsection{Ignition switch - resolving coupling in FRs}

In the beginning 2001-2002, some Saturn Ion ignition switches exhibited low torque during production testing. From mid-2004 thru late 2005, some Chevy Cobalt and Saturn Ion were reported to "inadvertently turn off" the ignition switches while in motion. From 2006 thru 2014, a series of investigations were undertaken to address the 
problem including a secret redesign in 2006. The activities culminated with a massive recall in early 2014 . For details and timeline of the events, see [5].

The primary issue was the unintended shifting out of the "Run" position that caused the engine to stall, knocking out airbags, power steering, and brakes. We describe below the configuration of the ignition switch, the physics that govern its operation and the flaw inherent in the design.

Figure 3 shows a photo of an ignition switch assembly. On one end of the assembly is the ignition switch and on the other end, the ignition key.

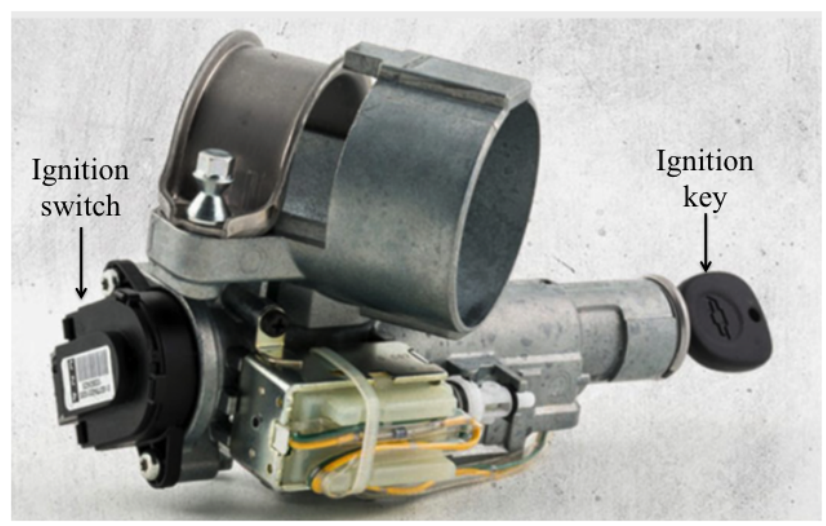

Fig. 3. Ignition switch assembly

Figure 4 shows the top view of the ignition switch. The ignition key rotates the ignition switch by means of a shaft through the slot shown.
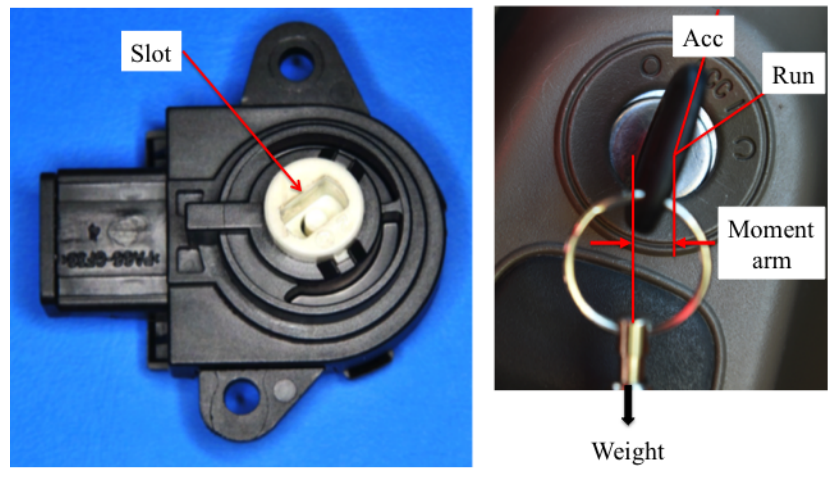

Fig. 4. Top view of ignition switch

Fig. 5. The key positions

Figure 5 shows the ignition key in the key cylinder at "Acc", the accessory position. With the key at this position, the engine is off. Another position of interest is the "Run" position at which the engine is on. In a key with wide slot, the weight of the keychain is at an offset to one side. The offset provides leverage for the weight of the keychain to produce a torque that tends to shift the key from Run to Acc thereby stalling the engine.

Figure 6 shows the components inside the ignition switch: the spring-loaded plunger and the switch disc with détentes. Note that the cam profile comprising the two détentes is symmetrical about line A-A equidistant from the two detente positions. For details, see [6].

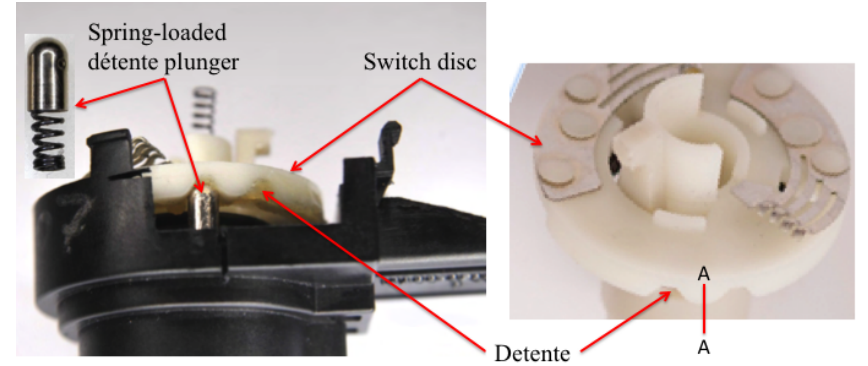

Fig. 6. Components inside the Ignition switch

When the ignition key is turned, it rotates the switch disc inside the ignition switch. As the switch disc with the détentes rotates and glides over the spring-loaded plunger, the plunger retracts out of the Run (Acc) détente and extends into the Acc (Run) detente to hold the switch in Acc (Run) position.

The FRs for the ignition switch are:

$\mathrm{FR}_{1}$ : Prevent unintended shifting from Run to Acc; the larger the resistance to shifting the better.

$\mathrm{FR}_{2}$ : Make the shifting from Acc to Run easy; the smaller the resistance to shifting the better.

Like the hubcap design, the two FRs are opposing. If the design cannot independently satisfy the two FRs, we once again would end up with conflict and compromise.

The resistance to shifting from one détente position to another is proportional to the energy needed to retract the plunger from one detente and extend it into the other. This energy may be derived as follows.

Referring to Figure 7, the cam profile that glides over the plunger is an inverted peak with two valleys. At one valley, the Run position, the plunger is deflected by an amount $\delta$. The associated energy stored is $\mathrm{k} \delta^{2} / 2$. As the cam glides over the plunger and the plunger retracts out of the détente to reach the "peak", the plunger is deflected by an amount $\Delta$. The associated stored energy is $\mathrm{k} \Delta^{2} / 2$. Thus, the energy "hump" to surmount the "peak" is $\mathrm{k} \cdot\left(\Delta^{2}-\delta^{2}\right) / 2$.

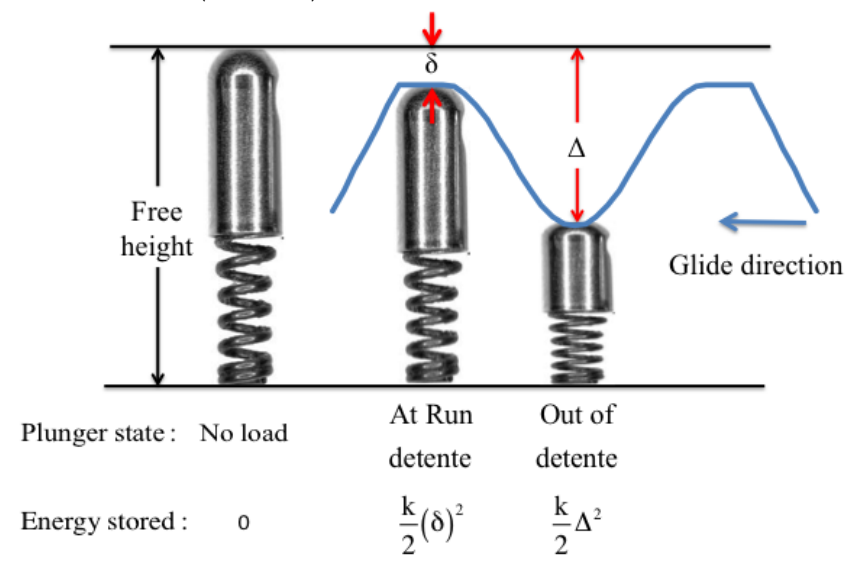

Fig. 7. Energy stored at various plunger positions, Run to Acc.

Since the cam profile is symmetric about the "peak" position, line A-A in Figure 6, the energy hump to surmount from Run to Acc is the same as that from Acc 
to Run, see Figure 8 below. Namely,

$$
\left[\begin{array}{c}
\text { Energy hump } \\
\text { Run } \rightarrow \text { Acc }
\end{array}\right]=\left[\begin{array}{c}
\text { Energy hump } \\
\text { Run } \leftarrow \text { Acc }
\end{array}\right]=\frac{\mathrm{k}\left(\Delta^{2}-\delta^{2}\right)}{2}
$$

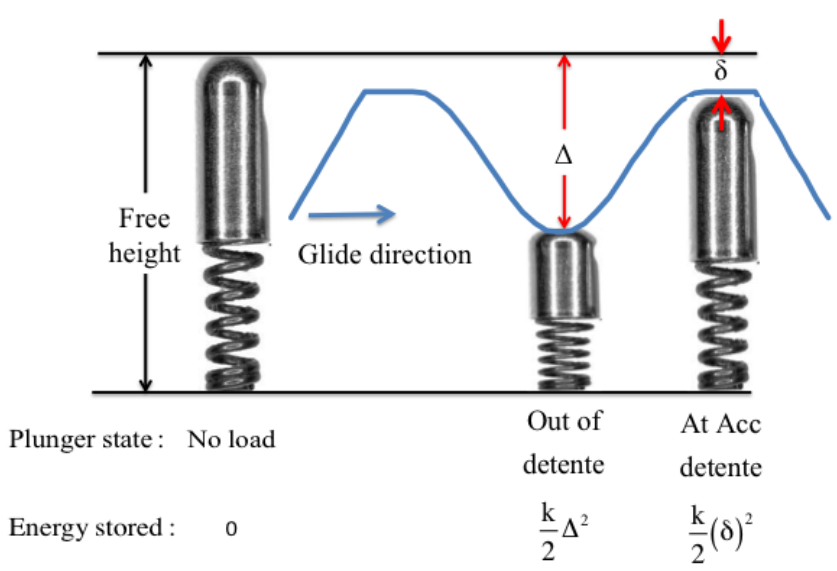

Fig. 8. Energy stored at various plunger positions, Acc to Run.

Given that resistance to shifting from Run (Acc) to Acc (Run) is proportional to energy hump to surmount, it follows that:

$$
\mathrm{FR}_{1}=\mathrm{FR}_{2}=\frac{\mathrm{k}\left(\Delta^{2}-\delta^{2}\right)}{2} .
$$

Thus the two FRs are functionally dependent on the same set of variables. Since $\mathrm{FR}_{1}$ and $\mathrm{FR}_{2}$ are opposing, the design cannot satisfy one FR without dissatisfying the other. This is the fundamental flaw of the design. To confirm functional dependency, we check if $|\mathbf{J}|=0$ :

$$
\begin{aligned}
{\left[\begin{array}{c}
\mathrm{FR}_{1} \\
\mathrm{FR}_{2}
\end{array}\right]=\left[\begin{array}{c}
\frac{\mathrm{k}\left(\Delta^{2}-\delta^{2}\right)}{2} \\
\frac{\mathrm{k}\left(\Delta^{2}-\delta^{2}\right)}{2}
\end{array}\right] } \\
{[\mathrm{J}]=\left[\begin{array}{ccc}
\partial \mathrm{FR}_{1} / \partial \mathrm{k} & \partial \mathrm{FR}_{1} / \partial \Delta \partial \mathrm{FR}_{1} / \partial \delta \\
\partial \mathrm{FR}_{2} / \partial \mathrm{k} & \partial \mathrm{FR}_{2} / \partial \Delta \partial \mathrm{FR}_{2} / \partial \delta
\end{array}\right] } \\
=\left[\begin{array}{ccc}
\frac{\left(\Delta^{2}-\delta^{2}\right)}{2} & \mathrm{k} \Delta & -\mathrm{k} \delta \\
\frac{\left(\Delta^{2}-\delta^{2}\right)}{2} & \mathrm{k} \Delta & -\mathrm{k} \delta
\end{array}\right]
\end{aligned}
$$

The three Jacobian determinants $|\mathrm{J}|$ that can be formed from two out of three columns in the $[\mathrm{J}]$ matrix above are all equal to zero.

$$
\left|\begin{array}{ll}
\frac{\left(\Delta^{2}-\delta^{2}\right)}{2} & \mathrm{k} \Delta \\
\frac{\left(\Delta^{2}-\delta^{2}\right)}{2} & \mathrm{k} \Delta
\end{array}\right|=\left|\begin{array}{ll}
\frac{\left(\Delta^{2}-\delta^{2}\right)}{2} & -\mathrm{k} \delta \\
\frac{\left(\Delta^{2}-\delta^{2}\right)}{2} & -\mathrm{k} \delta
\end{array}\right|=\mathrm{k}\left|\begin{array}{cc}
\Delta & -\delta \\
\Delta & -\delta
\end{array}\right|=0
$$

Therefore the two FRs are functionally interdependent.
In view of the functional dependency discussed, we now review the fixes that were made in the past. In 2006, there was a secret redesign involving changes in the plunger length - the free height - and spring stiffness, see Figure 9A. The changes increase the torque resistance to shift from Run to Acc, a desirable safety feature. Due to coupling, however, the changes also increase the torque resistance from Acc to Run, an undesirable customer satisfaction outcome. This coupling is indicated in Equation (5) in which any change in free height and spring stiffness, i.e., $(\delta, \Delta, \mathrm{k})$, to affect one FR invariably affect the other FR as well. In other words, the secret redesign did not address the fundamental flaw: the coupling of the two opposing FRs.

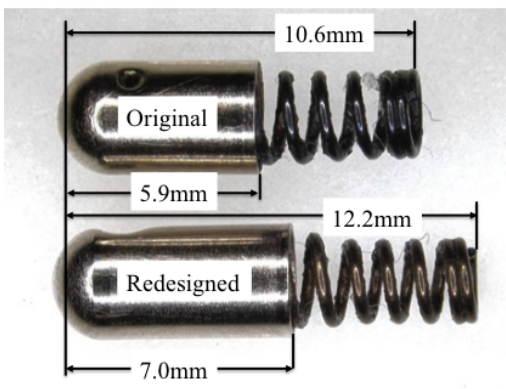

(A) Plunger redesigned

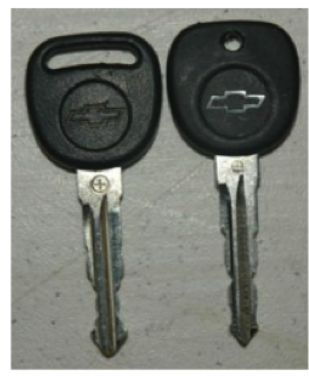

(B) Slot converted to hole
Fig. 9. Fixes for the defective ignition switch

Another remedy undertaken, which formed part of the recall effort, was to make the wide slot in the original key into a small hole; see figure 9B. This modification reduces the leverage for heavy key chains to rotate the key out of the Run position. The remedy is a good temporary fix. It reduces the potential torque input that rotates the key out of Run position. However, it still did not address the fundamental flaw of the functional coupling of the two opposing FRs.

As an illustration to removing the design fundamental flaw, one solution - albeit not the only one - is a cam profile shown in Figure 10. With this profile, the torque to shift the key out of Run position will be higher than the torque to shift out of Acc position.

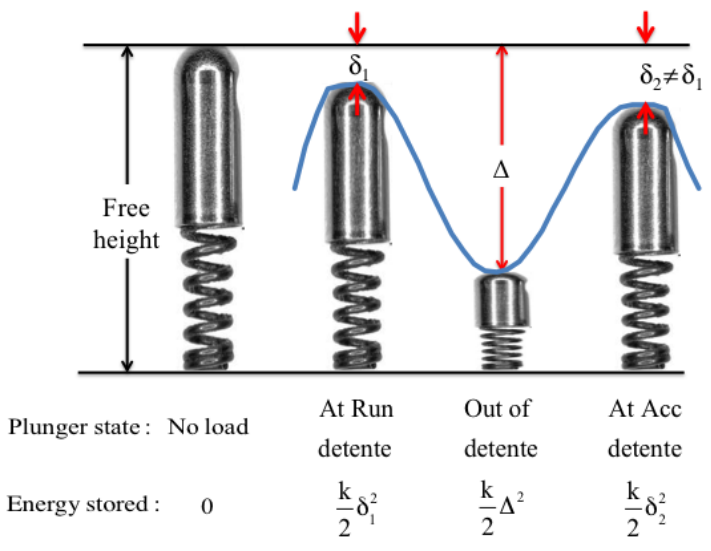

Fig. 10. A cam profile for generating unequal energy humps 
The FRs associated with the proposed cam profile are:

$$
\left[\begin{array}{c}
\mathrm{FR}_{1} \\
\mathrm{FR}_{2}
\end{array}\right]=\left[\begin{array}{c}
\frac{1}{2} \mathrm{k}\left(\Delta^{2}-\delta_{1}^{2}\right) \\
\frac{1}{2} \mathrm{k}\left(\Delta^{2}-\delta_{2}^{2}\right)
\end{array}\right] ; \quad \delta_{2} \neq \delta_{1} .
$$

The Jacobian matrix is:

$$
\begin{aligned}
{[\mathrm{J}] } & =\left[\begin{array}{cccc}
\partial \mathrm{FR}_{1} / \partial \mathrm{k} & \partial \mathrm{FR}_{1} / \partial \Delta & \partial \mathrm{FR}_{1} / \partial \delta_{1} & \partial \mathrm{FR}_{1} / \partial \delta_{2} \\
\partial \mathrm{FR}_{2} / \partial \mathrm{k} & \partial \mathrm{FR}_{2} / \partial \Delta & \partial \mathrm{FR}_{2} / \partial \delta_{1} & \partial \mathrm{FR}_{2} / \partial \delta_{2}
\end{array}\right] \\
& =\left[\begin{array}{cccc}
\frac{\left(\Delta^{2}-\delta_{1}^{2}\right)}{2} & \mathrm{k} \Delta & -\mathrm{k} \delta_{1} & 0 \\
\frac{\left(\Delta^{2}-\delta_{2}^{2}\right)}{2} & \mathrm{k} \Delta & 0 & -\mathrm{k}_{2}
\end{array}\right]
\end{aligned}
$$

All six Jacobian determinants $|\mathrm{J}|$ that can be formed from two out of four columns in the $[\mathrm{J}]$ matrix above are not equal to zero.

$$
\begin{aligned}
& \left|\begin{array}{cc}
\frac{\left(\Delta^{2}-\delta_{1}^{2}\right)}{2} & \mathrm{k} \Delta \\
\frac{\left(\Delta^{2}-\delta_{2}^{2}\right)}{2} & \mathrm{k} \Delta
\end{array}\right| \neq 0 ; \quad\left|\begin{array}{cc}
\mathrm{k} \Delta & -\mathrm{k} \delta_{1} \\
\mathrm{k} \Delta & 0
\end{array}\right| \neq 0 \\
& \left|\begin{array}{cc}
\frac{\left(\Delta^{2}-\delta_{1}^{2}\right)}{2} & 0 \\
\frac{\left(\Delta^{2}-\delta_{2}^{2}\right)}{2} & -\mathrm{k} \delta_{2}
\end{array}\right| \neq 0 ; \quad\left|\begin{array}{cc}
\mathrm{k} \Delta & 0 \\
\mathrm{k} \Delta & -\mathrm{k} \delta_{2}
\end{array}\right| \neq 0 \\
& \left|\begin{array}{cc}
\frac{\left(\Delta^{2}-\delta_{1}^{2}\right)}{2} & -\mathrm{k} \delta_{1} \\
\frac{\left(\Delta^{2}-\delta_{2}^{2}\right)}{2} & 0
\end{array}\right| \neq 0 ; \quad\left|\begin{array}{cc}
-\mathrm{k} \delta_{1} & 0 \\
0 & -\mathrm{k} \delta_{2}
\end{array}\right| \neq 0
\end{aligned}
$$

Thus the two opposing FRs are functionally independent of each other. They can be simultaneously satisfied without conflict and compromise.

\section{Concluding remarks}

The I J I criterion substantiates the Independence Axiom. It provides a formidable proof that non-compliance with the axiom will impede a design from fulfilling its functional requirements.

A design mistake, the hubcap, identified but not taught will reappear, as in the ignition switch, until the lesson is learned. The I $\mathbf{J} \mid$ criterion can serve as the mathematical basis for teaching and implementing Independence Axiom in design so that design mistakes need not be learned again and again.

\section{Disclosures}

The author was a member of a team that worked on the hubcap design. He was not involved in any way in the recall of the ignition switch. He was therefore not privy to information and data that went into the analyses and redesign for the recall. All the analyses and conclusions drawn by the author about the ignition switch herein were based on publicly available information and data.

\section{References}

1. N.P. Suh, The Principles of Design, (Oxford University Press, 1990).

2. H.L. Oh, Mathematical underpinnings for achieving design functional requirements, Proc. CIRP 50, pages 228-233, (2016).

3. H.L. Oh, Mathematical exposition of the design axioms, in A.M. Farid and N.P. Suh (eds), Axiomatic design in large systems: complex products, buildings and manufacturing systems, Chapter 2, Springer, (2016).

4. H.L. Oh, "Modeling Variation to Enhance Quality in Manufacturing." Conf. on Uncertainty in Engineering Design, NBS, (1988).

5. http://www.autonews.com/section/report01

6. https://docs.house.gov/meetings/IF/IF02/20140401/10 2033/HHRG-113-IF02-20140401-SD061.pdf 